\title{
WEGE DER DIFFERENZIERUNG IM FREMDSPRACHENUNTERRICHT
}

\author{
ANNA PERES, Hochschulprofessorin \\ EDUTUS-Universität \\ peres.anna@edutus.hu
}

DOI 10.47273/AP.2020.20.106-119

\begin{abstract}
ABSTRAKT
Im Zentrum des Artikels steht die Suche nach Wegen, wie man mit Hilfe des differenzierenden Unterrichts die Neugierde der Lernenden, die Sprache grundsätzlich selber zu erkunden und die innere Motivation, selbstständig Antworten auf die Fragen zu finden, aufbewahrt werden kann.

Potenziale freizulegen, selbstständiges Lernen zu ermöglichen, Wissen begeisternd zu vermitteln, die Kraft in der Vielfalt eines Teams aufzuzeigen sind Aspekte des Unterrichts, die erheblich dazu beitragen können, den Lernenden die Fremdsprache erlebnisorientiert beizubringen.

Wenn Klassenzimmer und die Alltagsrealität der Studierenden in den Aufgabenstellungen nicht streng auseinandergehalten sind, wird es ermöglicht, relevante Inhalte auch außerhalb des Unterrichts zu animieren und somit eine breite Palette der Differenzierungsmöglichkeiten aufzuzeigen.
\end{abstract}

\begin{abstract}
The differentiating teaching method allows us to sustain the students' curiosity so they can discover the language themselves, furthermore it allows us to maintain their motivation to independently find the answers to the questions.

Unlocking potential, enabling independent learning, imparting knowledge enthusiastically, demonstrating the strength in the diversity of a team are aspects of teaching that can make a significant contribution to teaching students the foreign language in an experience-oriented manner.

If the classroom and the everyday reality of the students are not strictly separated in the tasks, it is possible to animate relevant content outside of class and thus demonstrate a wide range of differentiation options.
\end{abstract}

\section{Einführende Gedanken}


Lernen und Lehren ist ein Prozess mit unebenen Kurven und schönen Ausflügen. Es liegt eben seinem Prozesscharakter zugrunde, dass man seinen eigenen Weg gehen kann, einmal mit eiligeren Schritten, ein anderes Mal langsamer. Als Lehrende seit über zwei Jahrzehnten kann ich überzeugt behaupten, das Schönste am gemeinsamen Weg die Begleiterrolle selbst ist: schafft man es, auf die individuellen Bedürfnisse der Lernenden einzugehen und zu erkennen, dass mit einem lobenden Wort mehr erreicht werden kann, als mit Kritik, dann ist viel dafür getan, motivierend maßgeschneiderte Unterstützung zu geben.

In diesem Sinne ist es nicht besonders angebracht, bei der Differenzierung über Fortgeschrittene und Schwächere, oder eben über Leistungsstarke und Leistungsschwache zu unterscheiden. Erst in Bezug auf den eigenen Lernweg lässt sich über starke, mittelstarke, etwas langsamere Fortschritte zu sprechen. Wörter, Fachwörter sind aber korpuskularem Charakter. Im Folgenden beziehen sich die genannten Kategorien erst der Einfachheit halber auf die Unterrichtssituation, im Klassenraum mit Differenzen erfolgreich umzugehen und die Lernenden bestmöglich fördern zu können.

\section{II. (Günstige) Voraussetzungen für die Differenzierung}

Eine bedeutende gesellschaftlich-bildungspolitische Errungenschaft der vergangenen Jahrzehnte ist, dass Kinder mit Lernstörungen im Unterrichtsprozess immer mehr mit Erfolg unterstützt werden können. Ohne die Bereitschaft, Bücher, Lernmaterialien zu entwickeln, entsprechende Methoden anzuwenden, um Leistungsvermögen nach einer breiteren Palette zu differenzieren, wäre es kaum möglich gewesen (vgl. Heimlich 2016: S. 20).

Lernstörung ist aber immerhin die extremste Variante der bunten Vielfalt, die die individuelle Besonderheit jedes einzelnen Lernenden beschreibt. Im Lernprozess funktioniert man ebenfalls nach seiner exklusiven Beschaffenheit: je nach Erfahrungen, Lerngeschwindigkeit, Motivation, Ausdrucksmittel, Strategien, Interessenkreis und Ziele etc. verfügt man über Bedürfnisse anderer Art.

Der diverse Charakter von Individualität und die Funktion der Motivation beim Lernen werden in der Studie von Andreas Krapp ganz exakt und bestens abgebildet:

„Doch hinter den scheinbar identischen deskriptiven Kategorien können sich ganz unterschiedliche psychologische Sachverhalte verbergen, die auf jeweils unterschiedlichen psychologischen Wirkmechanismen beruhen. Dies wird offensichtlich, wenn man z.B. das 
Gefühl des Wohlbefindens eines am Badestrand dösenden Urlaubers mit dem Wohlbefinden eines Bergsteigers vergleicht, der gerade die letzten Meter einer für ihn extrem schwierigen Klettertour bewältigt - oder eines Schülers, der nach einer längeren Phase des intensiven Nachdenkens und Ausprobierens endlich die richtige Lösung einer Mathematikaufgabe gefunden hat, weil er mit einem Mal das zugrundeliegende mathematische Prinzip verstanden hat. Ähnliches gilt für das Erleben intrinsischer Motivation. Sie wird auf der deskriptiven Ebene im Allgemeinen als ein motivationaler Zustand beschrieben, der den inneren Neigungen und Wünschen der handelnden Person entspricht und $\mathrm{zu}$ dessen Aufrechterhaltung keine extrinsischen Anreize (z.B. Lohn oder Strafe) erforderlich sind (Krapp 2005: S.638).“

Im Sinne der oben geschilderten Gründe gilt es, dass es im Prinzip keine homogene Gruppe gibt.

Für die erfolgreiche Durchführung differenzierender Methoden wird im Optimalfall als bestimmender Faktor eine motivierte Lehrkraft vorausgesetzt, der bereit ist, konzentriert die Aufmerksamkeit zu teilen, sich effektiv vorzubereiten und auf die individuellen Bedürfnisse der Lernenden einzugehen.

Bezüglich der Lernerseite sollte im Zeichen der Heterogenität eine Diagnose über die Stärken und Schwächen jedes einzelnen vorhanden sein, damit die Ziele bestmöglich personifiziert werden können.

Weitere ausschlaggebende Aspekte des Voraussetzungsfeldes der erfolgreichen Differenzierung sind die optimale Lernumgebung und die gemeinsam vereinbarte Angabe der minimalen Anforderungen. Und nicht zuletzt liegt der gemeinsamen Arbeit in der Lerngruppe selbstverständlich die Akzeptanz von Unterschieden zugrunde.

\section{Differenzierung - Rahmensatz}

Grundsätzlich lassen sich zwei Typen von Differenzierung unterscheiden: äußere und innere Differenzierung. Während die erste eine stärkere Rolle des Pädagogen voraussetzt, drückt sich im Falle der letzteren die Dominanz der Lernendenrolle in den Vordergrund.

„Von äußerer Differenzierung spricht man

a) bei der Zuordnung eines Schülerjahrganges in die verschiedenen Schulformen des mehrgliedrigen Schulsystems (z.B. Hauptschule, Gymnasium), b) wenn Schüler in 
fachspezifische Leistungsgruppen mit unterschiedlichen Anforderungen eingeteilt werden (z.B. in der Gesamtschule) oder c) wenn sie neben dem gemeinsamen Unterricht in den Pflichtfächern dann auf verschiedene Wahlpflichtfächer (z.B. auf der AHS-Oberstufe) aufgeteilt sind. Bei der äußeren Differenzierung bleiben die Schülergruppierungen im Allgemeinen in ihrer Zusammensetzung über einen längeren Zeitraum gleich. Die einzelnen Gruppen werden räumlich getrennt voneinander unterrichtet.

Innere Differenzierung ist hingegen meist (aber nicht ausschließlich) fachbezogen und betrifft alle didaktisch-methodischen Maßnahmen innerhalb eines gemeinsamen Klassenunterrichts, die darauf abzielen, den individuellen Begabungen, Fähigkeiten und Interessen der Schüler gerecht $\mathrm{zu}$ werden. Innere Differenzierung (manchmal wird für sie auch der Ausdruck Binnendifferenzierung verwendet) findet grundsätzlich in der heterogenen Lerngruppe statt. Sie will in bestimmten Lernbereichen, trotz unterschiedlicher Lernvoraussetzungen, möglichst alle Schüler unter Einsatz entsprechender Arbeitsweisen bzw. Arbeitsformen und Arbeitsmittel zur Beherrschung der als grundlegend erachteten Lernziele führen. Das kann in manchen Fällen bis zur Individualisierung des Unterrichts gehen. Dabei bewältigen Schüler einzeln oder partnerschaftlich mit nach Schwierigkeitsgrad entsprechend gestalteten Unterrichtsmaterialien unterschiedliche Lernwege zu gleichen Zielen. Sie können ihr Arbeitstempo dabei selbst bestimmen. Der Lernfortschritt wird am Erreichen operationalisierter Ziele laufend vom Lehrer (eventuell auch von den Schülern selbst) überprüft und Zurückgebliebene können Hilfen (vom Lehrer, von anderen Schülern, von Medien - z.B. vom Computer) in Anspruch nehmen. Die individuelle Lernphase dauert jedoch nur so lange, bis ein gemeinsames Lernen in der Klasse wieder sinnvoll ist“" (Sitte 2001: S. 199).

Die erhofften Ergebnisse der Differenzierung sind vor allem Chancengleichheit und die bestmöglich ideale Förderung jedes einzelnen. Die Unterstützung der Selbständigkeit und der Autonomie, sowie die Motivationsförderung sind dadurch nicht nur für den gegebenen Unterricht, sondern für die Formen sozialen und personalen Lernens im späteren ebenfalls ausschlaggebend.

Die differenzierenden Maßnahmen führen im Optimalfall zur Zunahme der kommunikativen Kompetenz, die konzentrisch wiederum weitere bestimmende Aspekte des Lernprozesses, wie z. B. die Konfliktbewältigungsfertigkeit, Kooperation, Einbezug und Stabilisierung der ganzen Lerngruppe, Vermeidung von Über-und Unterforderung, belebt (vgl. Bönsch 2009: S. 34). 
Der differenzierenden Aufgabenstellung liegen je nach Ausmaß zwei Kriterien zugrunde: das Kriterium der Vorgabe und das der Steuerung (Bönsch 2008).

In diesem Kontext werden nachgehende Differenzierung, Bearbeitungsdifferenzierung und freigebende Differenzierung unterschieden. In der ersten Form gilt es, den Unterricht gemeinsam anzufangen, um nachher Aufgaben und Übungen anzugeben, die nach Quantität (Zahl der Aufgaben), Qualität (Anspruchsniveau), Umfang der Unterstützungsleistung und Zeit unterschiedlich sind.

In der zweiten Form wird die Bearbeitungszeit angegeben, die flexibel und individuell eingeteilt werden kann. Die vorgegebenen Lernaufgaben sind mit Hilfe von Büchern, Lernmaterialien selbstständig auszuführen.

Bei der letzten Form, der freigebenden Differenzierung wird der zu lernende Stoff vom Lehrenden und den Lernenden gemeinsam aufgeteilt und nach einem vereinbarten Zeitplan selbstständig bearbeitet.

In der Fachliteratur können im Bereich DaF Typologien verschiedenster Art erforscht werden. Im Einklang von den Experten Kiper, Scholz und Kaufmann können in großen Zügen folgende Möglichkeiten der Binnendifferenzierung aufgestellt und jeweils durch unterschiedliche Sozialformen unterstützt werden (vgl. Kiper 2008, Scholz 2007, Kaufmann 2007):

- Niveaudifferenzierung: Differenzierung nach Leistungsanforderung oder Schwierigkeitsgrad

- Differenzierung nach Sprachniveaus

- Differenzierung nach Lernzielen

- Differenzierung nach Inhalten, Themen

- Differenzierung nach Lernmaterialien, Textsorten oder Medien

- Differenzierung nach Methoden (offen, bzw. geschlossen)

- Differenzierung nach Zeitrahmen

- Differenzierung nach Lerntechniken oder Lernstrategien (individuellen Lernstile)

- Differenzierung nach Aufgabentypen.

\section{Methoden: Differenzierung in der Praxis}

\section{Sanfte Formen der Differenzierung}

In der sogenannten latenten Form der Binnendifferenzierung (vgl. Wischer 2008) wird grundsätzlich gruppenmäßig gemeinsam gearbeitet, wobei gegebenfalls Einzelne gesondert 
angesprochen werden. D.h. im Frontalunterricht werden vom Lehrenden entweder individuelle Fragen gestellt, oder individuelle Korrekturen durchgeführt, bzw. individuelle Hilfestellungen gegeben. Eine weitere Lösung dabei ist, offene Fragen an die ganze Lerngruppe zu stellen und für die Lernenden frei möglich zu machen, wer sich wie einbringt.

Die geschilderten Möglichkeiten funktionieren jedoch erst in Gruppen erfolgreich, in denen die Heterogenität nicht allzu groß ist, und wenn sie vorwiegend in zielorientierten Lernsituationen eingesetzt werden.

\section{Einzelunterricht (innerhalb der Gruppe)}

Der Einzelunterricht in der Gruppe ist die radikalste Form der Binnendifferenzierung, indem hier die einzelnen Lernenden in Abstimmung mit dem Lehrenden je individuelle Lernziele für den eigenen Lernfortschritt formulieren. Im extremsten Fall ist ein jeder mit seinen eigenen Aufgaben nach angelegten Wochen- oder Monatsplänen einzeln beschäftigt, wobei vom Lehrenden maßgeschneiderte Unterstützung je nach Bedarf geleistet wird.

Darüber hinaus kann z. B. durch die Erstellung unterschiedlicher Arbeitsblätter ebenfalls ermöglicht werden, individualisierte Übungen für die eigenständige Durchführung anzubieten, oder mit Hilfe individueller Lernkarteien, Wörterbücher den eigenen Lernweg zu unterstützen. Die Portfolioarbeit mit einem individuellen Kompetenzraster kann dabei helfen, durch die angeordneten und gesammelten Dokumente, Übungen den Lernfortschritt zu belegen und bewusst zu verfolgen. Diese Formen des individualisierenden Lernens sind stark dadurch gekennzeichnet, „dass hier der Strukturierung und aktiven Rolle der Lehrenden eine zentrale Rolle zukommt“" (Wischer 2008: S. 716).

\section{Niveaudifferenzierung}

Bei der Differenzierungsform nach Leistungsanforderung oder Schwierigkeitsgrad macht der mittelstarke Gruppenbereich die implizite Niveaunorm aus, wobei differenzierend für die leistungsschwächeren, oder -stärkeren Lernenden extra Wahlmöglichkeiten angeboten werden. Fortgeschrittene können daher z. B. in Form von Schwerpunktaufgaben diverse Übungen erhalten, während der Großteil der Lerngruppe Übungsaufgaben durchführt. Um die Ergebnisse der Schwerpunktaufgaben und dadurch nützliche Informationen oder weitere relevante Kenntnisse mit den anderen zu teilen, können zusammenfassende Präsentationen in der Großgruppe gehalten werden. 
Nach einem anderen Lösungsweg können freie Aufgaben für Fortgeschrittene und zugleich konkrete Aufgaben für die anderen vorgegeben werden. Fortgeschrittene können in diesem Sinne beispielsweise frei zu einem bestimmten Thema einen Text formulieren, während die anderen Textbausteine bekommen oder einen bereits geübten Paralleltext schreiben.

Eine Variante dazu stellt das Hausaufgabenmodell dar, wobei die schwächeren oder langsameren Lernenden Zusatzangebote oder ergänzende Aufgaben außerhalb der gemeinsamen Kurszeit erhalten.

Das sogenannte Nachhilfemodell schließt optional verschiedenartige Realisationsmöglichkeiten ebenfalls außerhalb der gemeinsamen Kurszeit in sich, wie u.a. den kursbegleitenden Einzelunterricht, der von der Bildungseinrichtung selbst angeboten wird, oder die organisierten privaten Hilfen (z.B. die Vermittlung von Mentoren).

Die Bereithaltung einiger Reserveaufgaben kann dem Lehrenden in Situationen nützlich zur Seite stehen, um zu vermeiden, dass sich die besonders schnellen Lernenden langweilen könnten. Wichtig ist dabei, vorab zu definieren, welche Inhalte je nach Ziel bzw. Kontext des Kurses den Grund- oder Basisstoff bilden. Fundamente sind Ziele und Inhalte, die alle Gruppenmitglieder erarbeiten sollten, zusätzliche Aufgaben (Additum) sind ergänzende, freie Inhalte/Ziele für einzelne Gruppenmitglieder.

„In diesem Zusammenhang wird auch häufig die Differenzierung über quantitativ unterschiedliche (oder unterschiedliche schwierige) „Hausaufgaben“ angewendet. Eine logische Konsequenz ist auch die Verwendung differenzierter Kursunterlagen (z.B. in Form von Ringbüchern mit unterschiedlichen Einlagen oder als Skripten mit fakultativen Zusatzmaterialien)““(Aschemann 2011: S. 7.).

Niveaudifferenzierung kann durch diverse Formen von Teamteaching erheblich unterstützt werden. Wird die Großgruppe zumindest für den Übungsteil des Unterrichts in zwei Parteien, d.h. dauerhaft auf eine „Regelgruppe“ und eine „Fördergruppe“ (Aschemann 2011 S. 8.) aufgeteilt, kann eine zielgerechte Differenzierung ermöglicht werden. Dabei sollte im Sinne einer durchlässigen Struktur auf jeden Fall berücksichtigt werden, einen Gruppenwechsel durchgehend $\mathrm{zu}$ ermöglichen, um ein gesundes Selbstbild der schwächeren Schüler zu bewahren. Wege dazu sind in Lösungsformen zu finden, wo z. B. neben den zwei unterschiedlich leistungsstarken Untergruppen parallel abwechselnd in Kleingruppen, in Paararbeit und gemeinsamem Unterricht gelernt wird. 
Bei der Tischgruppenform wird ermöglicht, die fortgeschrittenen Lernenden bewusst herauszufordern, indem ihnen die Verantwortung übertragen wird: die Lernenden unterschiedlichen Wissens arbeiten jeweils gemeinsam an einem Tisch, wobei je ein Tischgruppensprecher gewählt ist.

Gruppenaufgaben, bei denen manche sehr aktiv und andere deklariert passiv sein können, geben Schwächeren die Möglichkeit, sich auf Basisinformationen zu konzentrieren, ohne sich dabei zu frustrieren. Beim Lesen eines Textes in Dreier-Gruppen kann z. B. ein Lernender den Text vorlesen, der andere auf den Textteil zeigen, der vorgelesen wird und der dritte unterstreicht schließlich die wichtigsten Schlüsselwörter (die Lernenden, die sich mehr Aufmerksamkeit bedürfen, können sich dabei bloß auf das Mitzeigen bzw. auf die Schlüsselwörter konzentrieren).

Wenn die Lernenden von Anfang an an differenzierenden Aufgabenstellungen gewohnt sind, sie etwa als einen normalen Zustand betrachten, können individuelle Zielsetzungen leicht angestrebt werden. Mit Hilfe unterschiedlicher Applikationen ist es möglich, dazu verschiedenartig individualisierte Arbeitsblätter mit Wortsuchrätseln, Schlangentexten und Lückentexten zu erstellen.

Fertigkeitsförderung sollte bestmöglich umgehend erfolgen. Lesefertigkeit z. B. kann in differenzierter Form gefördert werden, indem ein Text zuerst zum Lesen ausgegeben und danach mehrere Arbeitsblätter mit Verständnisfragen vorbereitet werden (z.B. ein Blatt mit einfachen Ja-Nein-Fragen, ein weiteres mit mittelschweren Fragen und eine dritte Version mit komplexen Fragen (vgl. Kaufmann 2007).

Schnippeltexte stellen ebenfalls eine ausgezeichnete Möglichkeit für Niveaudifferenzierung dar. Dabei wird ein Text wird gemeinsam gelesen und danach zerschnippelt. Ein Satz mit größeren Schnippeln kann natürlich leicht rekonstruiert werden, während ein Satz mit kleineren Schnippeln schwerer zusammenzusetzen ist und ein Satz, in dem sogar manche Schnippel fehlen (und so die Sätze ergänzt werden sollen) ist dabei am meisten schwierig (vgl. Kaufmann 2007). Nicht nur Sätze, sondern auch Volltexte können natürlich zerschnippelt werden.

Darüber hinaus können Lückentexte auch mit wenigem Aufwand zur differenzierten Bearbeitung angefertigt werden, indem ein Text mit mehr Lücken schwieriger, darum mit weniger Lücken natürlich leichter nachvollzogen werden kann. Noch leichter ist die Arbeit, wenn die einzufügenden Wörter extra vorgegeben werden (und noch leichter, wenn sie sogar in der richtigen Reihenfolge angeboten werden). 
Das eigenständige Erstellen von Unterrichtsmitteln erlaubt Fortgeschrittenen Lernenden, Übungsaufgaben, Themenhefte für Aufgabenstellung oder ein Quiz selber anzufertigen, die dann nach der Kontrolle des Lehrenden von den anderen in der Gruppe gemeinsam gelöst werden können.

Eine weitere Form der Niveaudifferenzierung ist, wenn Kleingruppen mit unterschiedlichen Materialien und Methoden parallel arbeiten. Die am meisten fortgeschrittenen Lernenden führen z. B. ohne Vorgabe eine Aufgabe (z. B. einen Lesetext) aus, während die zweite Gruppe die gleiche Aufgabe mit angegebenen Leitwörtern und Ausdrücken löst. Auf dem nächsten, dritten Niveau werden vorgegebene Satzteile zu einem Text geordnet und schließlich auf dem letzten Niveau arbeitet der Lehrende mit der Gruppe zusammen.

Nach Demmig (2008) ist die Dialogarbeit die klassische Form der Binnendifferenzierung im Sprachunterricht. Die vorher bewusst zusammengestellten Paare bekommen hier Dialoge zum Üben, die zielgemäß vorbereitet worden sind. Eine alternative Form stellt dazu die Fragensammlung zur gegebenen thematischen, lexikalischen oder grammatischen Einheit, die dann paarweise frei nach Wahl zu bearbeiten ist. Ähnlich funktionieren Bilderdominos oder auch Bildkarteien.

Qualitativ unterschiedliche Hausaufgaben dienen den Zwecken der Differenzierung ebenfalls ausgezeichnet. Fortgeschrittene können Fragen zu einem Text frei beantworten oder eigenständig Sätze bilden, sogar einen Text allein formulieren. Lernende mit etwas schwächerem Leistungsvermögen bekommen dafür einen Lückentext mit auf das Gelernte fokussierten Aufgabenstellungen.

\section{Offene Aufgabenstellungen}

Grundsätzlich geht es hier um Aufgaben mit qualitativ variablen Lösungs- oder Wahlmöglichkeiten. Die Lernenden können in diesem Kontext frei einen Lesetext für sich selbst aussuchen und bearbeiten oder von bereits angegebenen Fragen nur die für sich interessanten beantworten.

Offene Aufgaben mit quantitativ variablen Lösungsmöglichkeiten erlauben den Lernenden, wahlweise viel oder wenig dazu zu schreiben oder zu sagen. Die Aufgabe z. B., einen Text über die Zukunftspläne zu verfassen, ermöglicht Schwächeren, nur ein paar Sätze, Fortgeschrittenen, ausführlich viel dazu zu formulieren. 
Eine differenzierende Möglichkeit bieten Präsentationsaufgaben, wo je nach Wissensstand sehr verschiedenartige Leistungen nach sprachlicher Komplexität akzeptiert werden können. Alternativ ist auch das gemeinsame Verfassen eines kurzen Textes mit anschließendem Vorlesen angebracht, das vorher zu zweit geübt werden kann.

Der Projektunterricht mit einem umfassenden Thema, das in Gruppen je nach eigenem Interesse unterthemenartig bearbeitet wird, ermöglicht in seiner offenen Form auch eine breite Palette akzeptabler Lösungen.

Besonders attraktiv ist die Lernform in Lernstationen. Im Klassenraum werden Stationen mit Aufgaben und Unterrichtsmaterialien aufgestellt, welche von den Lernenden je nach Interesse oder Fähigkeiten oder Lerntyp nacheinander ausgeführt oder einfach geübt werden. Dabei kann es Pflicht- und Freistationen geben.

Der Ausgangspunkt in der Freiarbeit ist der Zeitrahmen. Die Lernenden sind mit Aufgaben und Unterrichtsmaterialien selbst beschäftigt, Tempo und Sozialform, eventuell auch Material können sie für sich selbst dabei aussuchen.

\section{Kooperative Differenzierung}

Lern- oder Sozialformen, die dem Zwecke der Kollaboration dienen, sind „Gruppenarbeit, bei der die Lernenden gemeinsam eine Aufgabe lösen, Partnerarbeit, bei der die Lernenden Gedanken austauschen, einander befragen oder miteinander üben, Lernerkorrekturen, bei denen die Teilnehmenden die Texte anderer Lernenden analysieren und kommentieren, Brainstorming, bei dem die Lernenden Ideen zu einem bestimmten Thema sammeln, PuzzleAktivitäten, bei denen jeder Lernende einen anderen Wissensaspekt beisteuert, Gemeinschaftliches Schreiben, bei dem eine Gruppe von Lernenden zusammen einen Text erstellt, z.B. einen Ratgeber (...), Gemeinschaftsprojekte, bei denen die Lernenden einen Aspekt ihrer Umgebung erkunden und später im Kurs darüber berichten (...), GruppenposterPräsentationen, in denen eine Gruppe von Lernenden zu einem bestimmten Thema oder Problem ein Poster entwirft usw.“(Kaufmann 2007: S.198).

\section{Abschließende Gedanken}

Die ausgeführten differenzierenden Ideen, Methoden, die im Klassenzimmer erlauben, demokratisch und mit guten Voraussetzungen eine Fremdsprache zu beherrschen, sind bewährte Katalysatoren im Lernprozess. 
Zieht man noch in Anbetracht, dass die Fragen, auf die im Unterricht Antwort gesucht wird oder Inhalte, die durchzuarbeiten sind, im Grunde praktisch ausschließlich vom Lehrenden gestellt, bzw. bestimmt werden. Die Erfahrung zeigt aber, dass die Motivation erheblich gestärkt werden kann, wenn die Lernenden ihre Welt, ihre Interessen mit in die Klasse bringen dürfen. Die Formen der kooperativen Differenzierung sind insbesondere geeignet, die Grenzen des klassischen Klassenraums zu sprengen, die schulische und private Wirklichkeit aufeinander $\mathrm{zu}$ beziehen, wo es verwirklicht werden kann, dass die Lernenden selbst die Fragen stellen können, die einen zentralen Platz in den Unterrichtseinheiten bekommen.

Die Stundenzahl ist begrenzt, Lernende und Lehrende sind oft überbelastet. Es ist nicht einfach, Zeit und Energie zu investieren, die Lernenden bezüglich ihrer Ziele, Lernstile, Persönlichkeit, Motivation, Vorwissen usw. möglichst genau kennenzulernen, beziehungsweise individuelle Förderungswege zu finden. Auf der anderen Seite der Waage stehen aber glückliche, entspannte Sprachbenutzer, Erlebnislernen und eine optimale Entfaltung der persönlichen Fähigkeiten. 


\section{Literatur}

1. Dr. Birgit Aschemann (unter Mitarbeit von Mag.a Petra Gugler und Mag.a Maria Nimmerfall): Vierzig Wege der Binnendifferenzierung für heterogene LernerInnenGruppen. Ein didaktischer Reader verfasst im Rahmen des Projekts „Deutsch und andere Erstsprachen im gemeinsamen Alphabetisierungskurs“. Frauenservice Graz 2011. http://www.aschemann.at/wp-

content/uploads/2015/05/Vierzig_Wege_Binnendifferenzierung.pdf (Herunterladen am 16-ten Juli 2020).

2. Bönsch, Manfred (2008): Methodik der Differenzierung. In: Die Berufsbildende Schule $60 / 2008$.

3. Bönsch, Manfred (2009): Erfolgreiches Lernen durch Differenzierung im Unterricht. Westermann Verlag, Braunschweig

4. Böttinger, Anja (2019): „Hilfe, mein Kurs ist so heterogen.“ - Methodentipps für den binnendifferenzierenden Alphabetisierungsunterricht. In: ALFA-Forum / Ausgabe 95 Sommer 2019.

https://www.iik.berlin/sites/default/files//publikationen/AF\%2095_Böttinger_2019_3.pdf (Herunterladen am 15-ten Juli 2020).

5. Demmig, Silvia (2008): Binnendifferenzierung und Heterogenität. In: Deutsch als Zweitsprache 2008/4. S. 34-39.

6. Demmig, Silvia (2007): Das professionelle Handlungswissen von DaZ-Lehrenden in der Erwachsenenbildung am Beispiel Binnendifferenzierung. München: Ludicium Verlag.

7. Dergovics, Elke (2010): Verschiedene Menschen, verschiedenen Sprachen - ein Kurs. In: Isop Gmbh; Rath, Otto; Hahn, Mariella (Hg.): Zwischenbilanz. Die Basisbildung in Österreich in Theorie und Praxis. S. 30-35. Online verfügbar unter: http://www.zukunftbasisbildung.at/Downloads/Situation/1-04-Dergovic.pdf (2010-10-21). (Herunterladen am 10-ten Juli 2020).

8. Ewers, Heike (2002): Maßgeschneidert oder von der Stange? Gruppenarbeit und Binnendifferenzierung. In: Passwort Deutsch. Didaktische Beiträge. Online verfügbar unter: http://www.passwortdeutsch.de/lehren/unterrichtstipps/download/binnendifferenzierung.pdf (2010-01-04).

9. Freiling, Elke; Biloa Onana, Marie; Sonntag, Ilse (2010): Binnendifferenzierung: gezielt und wohldosiert. In: Alpha-Forum 74/2010, S. 25-27.

10. Heimlich, Ulrich [Hrsg.]; Kahlert, Joachim [Hrsg.]; Lelgemann, Reinhard [Hrsg.]; Fischer, Erhard [Hrsg.]: Inklusives Schulsystem. Analysen, Befunde, Empfehlungen zum bayerischen Weg. Bad Heilbrunn : Verlag Julius Klinkhardt 2016, 159 S. - (KlinkhardtFforschung)

11. Hubertus, Peter (2010): Teilnehmerorientierung und das Verhältnis von Lehren und Lernen. In: Alpha-Forum 74/2010, S. 38-40. 
12. Károly, Krisztina szerk.]; Homonnay, Zoltán [szerk.] (2017): Diszciplínák tanítása -a tanítás diszciplínái. Mérési és értékelési módszerek az oktatásban és a pedagógusképzésben. Diszciplínák tanítása - a tanítás diszciplínái 5. kötet. ELTE Eötvös Kiadó.

13. Kaufmann, Susan (2007): Heterogenität und Binnendifferenzierung im DaZ-Unterricht. In: Fortbildung für DaZ-Kursleitende. Hueber 2007. S. 186 - 214.

14. Kiper, Hanna (2008a): Zur Diskussion um Heterogenität in Gesellschaft, Pädagogik und Unterrichtstheorie. In: Kiper, Hanna; Miller, Susanne; Palentien, Christian; Rohlfs, Carsten (Hg.) (2008): Lernarrangements für heterogene Gruppen. Bad Heilbrunn: Verlag Julius Klinkhardt. S. 78-105.

15. Kiper, Hanna (2008b): Unterrichtsplanung für heterogene Lerngruppen. In: Kiper, Hanna; Miller, Susanne; Palentien, Christian; Rohlfs, Carsten (Hg.) (2008): Lernarrangements für heterogene Gruppen. Bad Heilbrunn: Verlag Julius Klinkhardt. S. 127-152.

16. Konietzko, Gerd; Dahlmann, Monika (2004): Think-Pair-Share. In: Heterogenität. Unterschiede nutzen -Gemeinsamkeiten stärken. (Friedrich Jahresheft 22/2004). S.118119.

17. Kossmeier, Elisabeth (2009): Einzelnen gerecht werden. Linz: Pädagogische Hochschule Oberösterreich.

18. Krapp, Andreas (2005): Das Konzept der grundlegenden psychologischen Bedürfnisse. Ein Erklärungsansatz für die positiven Effekte von Wohlbefinden und intrinsischer Motivation im Lehr-Lerngeschehen. Zeitschrift für Pädagogik 51 (2005) 5, S. 626-641.

19. Kress, Karin (2016): Binnendifferenzierung in der Sekundarstufe - Das Praxisbuch. Auer Verlag, Augsburg.

20. Kuty, Margitta (2009): Binnendifferenzierung in Aktion. Einsteigertipps aus der Praxis. In: Praxis Fremdsprachenunterricht 6/2009, 3, S. 16-21.

21. Palmsdorfer, Brigitte (2006): Differenzierung KONKRET - Aus der Praxis für die Praxis. Ein Handbuch für die Grundschule. Wien: Jugend und Volk.

22. Plutzar Verena (2007): Empfehlungen für das Lehren in der Zweitsprache. Online verfügbar unter: http://www.integrationshaus.at/cgi-bin/file.pl?id=205 (2010-10-21).

23. Posch, Claudia (2010): Implikationen der gemeinsamen Alphabetisierung von Migrantinnen und Migranten und Menschen deutscher Muttersprache. Unveröffentlichte Bachelorarbeit, Karl Franzens-Universität Graz.

24. Salner-Gridling, Ingrid (2009): Querfeldein: individuell lernen - differenziert lehren. Wien: Amedia (im Auftrag des bm:ukk). Online verfügbar unter: http://www.bmukk.gv.at/medienpool/18764/mat_querfeldein.pdf (2010-10-21).

25. Scheunpflug, Annette (2008): Lernen in heterogenen Gruppen - Möglichkeiten einer natürlichen Differenzierung. In: Kiper, Hanna; Miller, Susanne; Palentien, Christian; 
Rohlfs, Carsten (Hg.): Lernarrangements für heterogene Gruppen. Bad Heilbrunn: Verlag Julius Klinkhardt. S. 66-77.

26. Scholz, Ingvelde (2007): Es ist normal, verschieden zu sein - Unterrichten in heterogenen Klassen. In: Scholz, Ingvelde (Hg.): Der Spagat zwischen Fördern und Fordern: Unterrichten in heterogenen Klassen. Göttingen: Vandenhoeck \& Ruprecht, S. 7-23. Online verfügbar unter:

http://www.pedocs.de/volltexte/2010/1450/pdf/Scholz_Spagat_Scholz_Normal_W_D_A. pdf (2010-10-21).

27. Schweckendiek, Jürgen (2001): Spiele und Spielerisches. Zur Förderung der Gruppenintegration und zur Binnendifferenzierung. In: Fremdsprache Deutsch, Heft 25/2001, S. 9-19.

28. Sitte, W.; Wohlschlägl, H. Hrsg. (2001): Beiträge zur Didaktik des „Geographie und Wirtschaftskunde"-Unterrichts. Wien, 564 Seiten (= Materialien zur Didaktik der Geographie und Wirtschaftskunde. Bd. 16) Institut für Geographie und Regionalforschung der Universität Wien

29. Wischer, Beate (2008): „Binnendifferenzierung ist ein Wort für das schlechte Gewissen des Lehrers". In: Erziehung und Unterricht, 2008/9-10, S. 714-722. Online verfügbar unter: http://www.oebv.at/sixcms/media. php/504/wischer.pdf (2010-10-21). 